Systematic Review

\title{
A Systematic Review of Single-Case Research on Science-Teaching Interventions to Students with Intellectual Disability or Autism Spectrum Disorder
}

\author{
Georgia Iatraki *(D) and Spyridon-Georgios Soulis \\ Department of Primary Education, University of Ioannina, 45110 Ioannina, Greece; ssoulis@uoi.gr \\ * Correspondence: g.iatraki@uoi.gr
}

check for updates

Citation: Iatraki, G.; Soulis, S.-G. A Systematic Review of Single-Case Research on Science-Teaching Interventions to Students with Intellectual Disability or Autism Spectrum Disorder. Disabilities 2021, 1, 286-300. https://doi.org/10.3390/ disabilities1030021

Received: 22 May 2021

Accepted: 14 September 2021

Published: 19 September 2021

Publisher's Note: MDPI stays neutral with regard to jurisdictional claims in published maps and institutional affiliations.

Copyright: (c) 2021 by the authors. Licensee MDPI, Basel, Switzerland This article is an open access article distributed under the terms and conditions of the Creative Commons Attribution (CC BY) license (https:// creativecommons.org/licenses/by/ $4.0 /)$.

\begin{abstract}
The present study records and evaluates science-teaching interventions for students with intellectual disability (ID) or Autism Spectrum Disorder (ASD) at all education levels, for better access to the general curriculum. We conducted a systematic review of the primary research literature published from 2008 to 2018. A total of 24 single-case designs were identified through determined inclusion criteria. The articles were coded based on specific variables, and they were analyzed as a descriptive synthesis. A synthesis was then approached and the PND effect size was calculated. The study concludes that using systematic instruction with supplemental practices and technology support in teaching science to students with ID or ASD will allow them to make more meaningful natural-world connections through their learning. In addition, designing postsecondary programs for this population will contribute to building a society with equal participation and progress. The necessity for continuous research in teaching science to students with ID or ASD is also emphasized.
\end{abstract}

Keywords: instruction; science; intellectual disability; Autism Spectrum Disorder (ASD)

\section{Introduction}

The need for understanding natural-world and science concepts has led researchers and professionals to design effective teaching interventions in science classrooms [1-4]. Science content knowledge and scientific ways of thinking are highlighted in the NGSS Legislation and Standards [5] to be crucial for all students, with or without disabilities, for them to access general science classrooms and participate equally in society while dealing with topics related to science [6].

\subsection{Teaching Science to Students with Intellectual Disability or Autism Spectrum Disorder}

The current review focuses on science-teaching interventions for students with intellectual disability (ID) or/and Autism Spectrum Disorder (ASD), due to the similarity of challenges that these students face. The definitions for both diagnoses are clearly differentiated, therefore teachers and practitioners consider instructional methods and supplementary techniques to support student academic performance. Intellectual disability is a neurodevelopmental disability that is characterized by significant limitations to intellectual functioning and adaptive behavior [7]. Intellectual disability appears before the age of 18 and includes deficits in conceptual, social, and practical skills. ASD is a neurodevelopmental disorder associated with difficulties in social communication and restrictive behavioral patterns [7].

The combination of academic and functional skills based on student needs, supports student social participation [8-10]. An important component of the learning process is knowledge that targets functional skills, as well as daily life skills. High levels of difficulty (e.g., memory deficits, lack of concentration, and barriers in academic content) experienced by students with ID or ASD result in lower achievement compared to their peers without 
disabilities [11,12]. Recent educational planning highlights student personal interests, their experiences, and several emotional factors that influence their efforts [13].

The literature indicates communication barriers, as well as cognitive deficits that negatively affect the participation and progress of students with ID or ASD in science [14]. Despite the emphasis on teaching content, there is little evidence surrounding how to teach science content to this population $[13,15]$. In particular, the literature emphasizes the limited interest of students with disabilities, because of traditional teaching approaches in the classroom [16,17]. Therefore, teachers should focus on basic science knowledge and understanding, and motivate students with ID or ASD to remain on task [18,19].

The implementation of systematic instruction is considered effective to include students (i.e., students with ID or ASD) in the classroom. Other supplemental teaching techniques, such as the system of least prompts, task analysis, time delay, and peer teaching, contribute to the effective learning of students with ID or ASD. Technology use provides additional motivations and learning experiences (i.e., visual representations), which increases student interest $[2,3,15,20,21]$.

Although studies of science-teaching interventions for students with ID or ASD are limited, their findings suggest that adapted procedures are required for student participation and achievement in the classroom [22]. Most curriculum reformers agree on the implementation of different principles in the science content curriculum, including a basic understanding of the natural world (e.g., physical phenomena) $[2,18,23]$. According to previous studies of science teaching, a positive effect of content learning on the behavior of students with ID or ASD was found [24]. Scruggs and Mastropieri [25] observed that science courses specifically designed for students with mild ID showed positive learning outcomes regarding adapted material and the design of relevant conclusions derived from teacher guidance. The effectiveness of mnemonic strategies for facilitating verbal communication for science concepts and physical phenomena was also noted in the classroom [24,25].

Despite the increase of empirical research in general academic content, such as reading and mathematics, a gap remains in the provision of academic content in science to students with ID or ASD [26]. Abstract concepts and demanding equation skills seem to present barriers in science teaching, especially in physics content. According to previous reviews [17,27], researchers agree that students can benefit from systematic instruction with supplemental combined teaching strategies. An interesting field that has emerged is science instruction related to technology and science instruction via inquiry learning. Spooner's review [17] considered including coding studies as "acceptable" via Horner's quality indicators, to identify effective interventions [28]. Researchers underlined that students with ID or ASD can evaluate science skills aligned with the general curriculum. Rizzo and Taylor [29] analyzed the bibliography and reviewed the teaching strategies and inquiry learning for students with several disabilities. They included 12 studies between 1992 and 2013 and concluded that the scientific vocabulary can serve as a self-monitoring strategy for problem-solving skills.

In a recent review by Apanasionok et al. [30], six electronic databases were searched, and 30 studies were examined. The studies focused both on student academic achievement and the assessment of students, and instructor experiences. The targets were science vocabulary and concepts, comprehension, and inquiry skills. The findings indicated that elements of systematic instruction contribute to successful science teaching for students with ID or ASD, but further research is needed. Knight et al. [31] synthesized 12 studies conducted between 2009 to 2018 addressing science content for students with ID and ASD. The main purpose of their review was to evaluate methodologically sound studies by meeting the quality indicators of the Council of Exceptional Children. Another review of research in science instruction was conducted by Taylor et al. [32] which involved students with ASD. The researchers included published studies between 2000 to 2018 and highlighted the importance of enhancing the science literacy of students with ASD for further progress in the classroom and participation in society. Following this trend, more research is needed on science academic content for students with ID and ASD with 
co-occurring ID, since they are usually enrolled in the same settings and supported by similar interventions that seem to be helpful and beneficial for them.

\subsection{The Present Study}

The current study was designed to investigate science-teaching interventions and criteria that contribute to the academic achievement of students with ID or ASD. Additionally, social validity could be assessed as a new variable in this research field. Our purpose is to identify, examine, and synthesize the initial outcomes of the included studies, as a comprehensive synthesis. We highlighted science vocabulary and comprehension science skills regarding science concepts for students with ID or ASD to access academic content aligned with the general curriculum. The results could support all graded educational programs and promote inclusion $[33,34]$. They could also be helpful to teachers and special educators [35]. The research questions were:

RQ1. What types of intervention strategies for supporting students with ID or ASD in science have been studied by researchers?

RQ2. Which educational objectives have been addressed and in which setting have they occurred?

RQ3. How effective were the interventions?

\section{Methods}

\subsection{Data Sources}

An extensive search of the literature was conducted during 2019 to retrieve studies that met inclusion criteria for this review. The following electronic databases were searched: (a) Educational Resources Information Center, (b) ScienceDirect, (c) Scopus, and (d) Google Scholar. The search terms used were instruction, science, intellectual disability, and autism spectrum disorder. Additionally, a list of combination of terms was used (e.g., teaching, learning, intervention, physics, chemistry, biology, geography, mental retardation, down syndrome, moderate intellectual disability, and autism) to identify the research base of academics and students with ID or/and ASD. The initial search yielded 1044 articles. An ancestral search was conducted through handsearching of prominent special education journals, such as Focus on Autism and Other Developmental Disabilities Education and Training in Autism and Education and Training in Autism and Developmental Disabilities to ensure we identified all studies matching our criteria. Finally, 63 additional studies were included.

\subsection{Study Selection}

The studies needed to meet the following inclusion criteria: (a) publication dates were in the specified scope of 2008-2019 in a peer reviewed journal in English, (b) participants were identified as having a diagnosis of ID or ASD with co-occurred ID and were in primary, secondary, or postsecondary education, (c) the study included an experimental design, (d) the independent variable was an intervention based on science vocabulary or basic comprehension of science content, and (e) the dependent variable was the assessment of science content.

The criterion referred to the experimental design, which led to single-case research design, which supports educational practices in targeted analysis of the individual as each participant serves as their own control. Performance prior to intervention is compared to performance during and/or after intervention. Therefore, synthesis procedures could help determine whether an intervention is effective in including change regarding performance [28]. Detailed characteristics of the studies included in the review are presented in Figure 1.

After records were identified and duplicates removed, the original search resulted in 1107 documents. Titles and abstracts of all documents were screened to determine whether they met inclusion criteria. As a result of the initial screening, 961 documents were excluded, resulting in a total of 146 documents that were screened by full text. Then, the authors reviewed the articles to ensure they met the final inclusion criteria. Following 
the screening, 122 full-text articles were excluded as duplicates (76), group studies (4), qualitative studies (2), reviews (13) and studies that did not meet the participant and publication criteria (27). The two coders came to an agreement on whether to include articles. A total of 24 studies conducted in the USA met the inclusion criteria and were consequently included in this review.

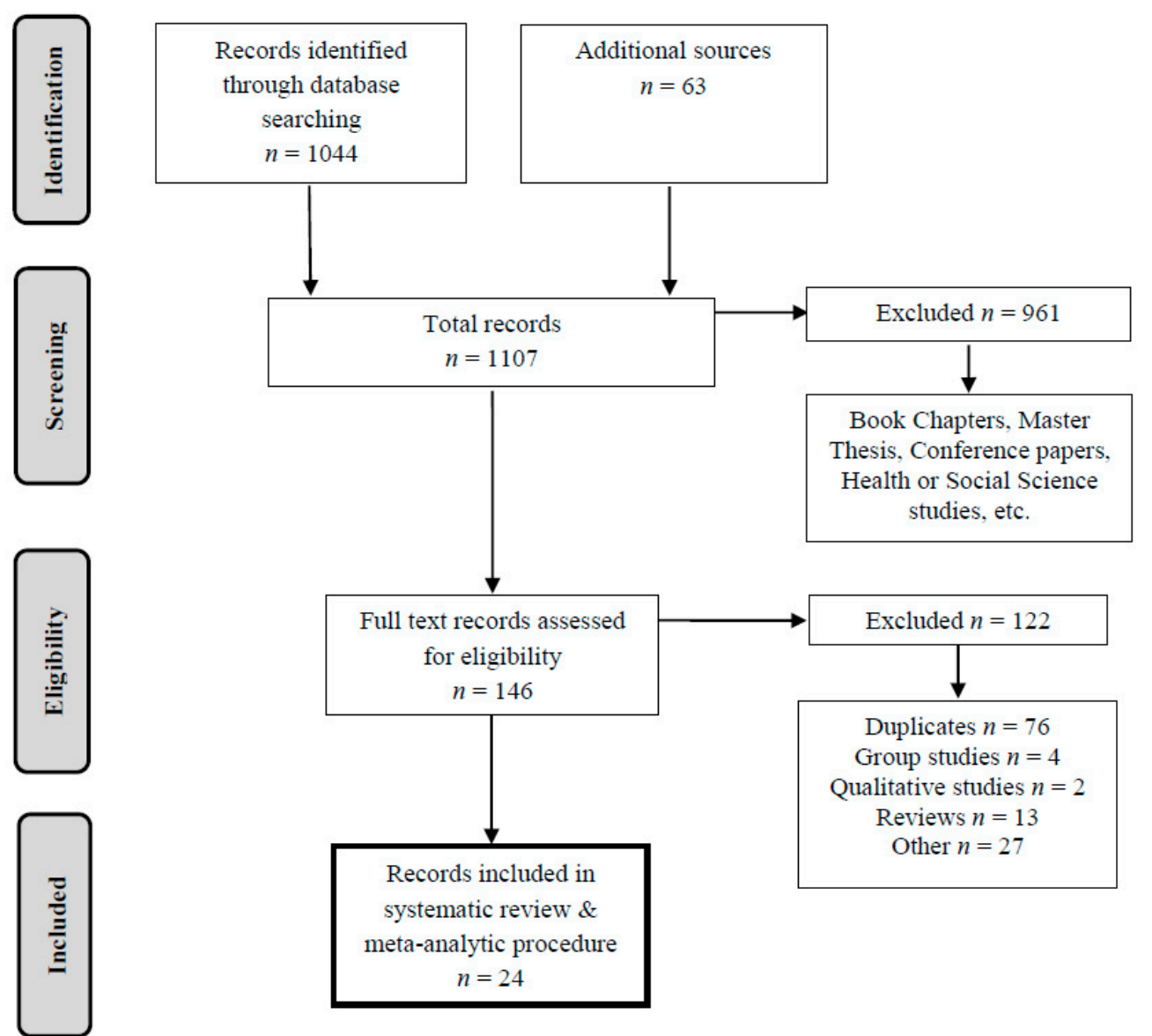

Figure 1. PRISMA flowchart of search strategy (Moher et al.) [36]; www.prisma-statement.org (accessed on 22 May 2021).

\subsection{Data Extraction and Coding}

A coding guide was created to extract relevant information to examine accessibility in science classrooms [37]. Data were extracted in an Excel Microsoft Office protocol and were categorized into three categories: (a) source description, (b) research methods and procedures, and (c) substantive issues. Source description data included publication date, author team, journal, and type of manuscript. Research methods and procedures included the type of research design, intervention components (baseline, intervention, generalization, maintenance), and measures used. Substantive issues included the variables implementer, problem types, instructional setting, disability category, participants' ages, and fidelity of implementation.

The descriptive analysis included detailed descriptions for the participants (number, age, gender, disability), setting and instructors, the effects of the independent variables on the dependent variables, science academic content and targets, supplemental teaching methods, research design, and social validity. See Tables 1 and 2 for the additional results of the synthesis of the reviewed research. 
Table 1. Synthesis of the studies.

\begin{tabular}{|c|c|c|c|c|c|}
\hline Citation & Participants & Age (or Grade) & $\begin{array}{l}\text { Setting/ } \\
\text { Instructor }\end{array}$ & $\begin{array}{l}\text { Science Content/ } \\
\text { Teaching Targets }\end{array}$ & $\begin{array}{l}\text { Intervention } \\
\text { Design }\end{array}$ \\
\hline Britton et al. [38] & $\begin{array}{c}1 \mathrm{M} \\
\text { Origin- } \\
\text { ID, IQ: } 51\end{array}$ & 17 & $\begin{array}{c}\text { RR } \\
\text { Paraprofessionals } \\
\text { peers }\end{array}$ & $\begin{array}{c}\text { Environment } \\
\text { (recycling, pollution) }\end{array}$ & $\begin{array}{l}\text { TA, TD } \\
\text { MPD }\end{array}$ \\
\hline Ciullo et al. [39] & $\begin{array}{c}1 \mathrm{M} \\
\text { Hispanic } \\
\text { ID, IQ: - }\end{array}$ & (4th grade) & RR, SE & $\begin{array}{l}\text { Biology (sea world) } \\
\text { C (concept maps) }\end{array}$ & $\begin{array}{c}\text { SI, prompting } \\
\text { Tech } \\
\text { (digital organizers) } \\
\text { MPD }\end{array}$ \\
\hline Collins et al. [40] & $\begin{array}{c}2 \mathrm{M} 2 \mathrm{~F} \\
\text { Hispanic } \\
\text { African } \\
\text { American } \\
\text { ID, IQ: } 62-71\end{array}$ & $16-19$ & Researcher & $\begin{array}{c}\text { Biology } \\
\text { (photosynthesis) } \\
\text { V, C }\end{array}$ & $\begin{array}{l}\text { TA and prompting } \\
\text { MPD }\end{array}$ \\
\hline Courtade et al. [41] & $\begin{array}{c}4 \mathrm{M} 4 \mathrm{~F} \\
\text { African } \\
\text { American } \\
\text { Caucasian } \\
\text { Hispanic } \\
\text { ID, IQ: } 39-54\end{array}$ & $11-15$ & $\begin{array}{c}\mathrm{GC}, \mathrm{T} \\
\text { paraprofessionals }\end{array}$ & $\begin{array}{c}\text { Physics (magnetism) } \\
\text { V }\end{array}$ & $\begin{array}{c}\text { IL, } \\
\text { prompting and TA } \\
\text { MPD }\end{array}$ \\
\hline Evmenova et al. [42] & $\begin{array}{c}4 \mathrm{M} \\
\text { Caucasian, } \\
\text { Hispanic } \\
3 \text { ID, } 1 \text { ASD, IQ }\end{array}$ & $16-20$ & $\begin{array}{c}\mathrm{GC}, \mathrm{T} \\
\text { paraprofessionals }\end{array}$ & $\begin{array}{c}\text { Environment } \\
\text { (decision and } \\
\text { navigation skills) } \\
\text { C }\end{array}$ & $\begin{array}{c}\text { Prompting } \\
\text { Tech } \\
\text { (interactive videos) } \\
\text { MPD }\end{array}$ \\
\hline Fetko et al. [43] & $\begin{array}{c}2 \text { M } 1 \text { F } \\
\text { Origin- } \\
2 \text { ID, } 1 \text { ASD } \\
\text { IQ: - }\end{array}$ & $12-14$ & $\begin{array}{c}\text { RR, } \mathrm{T} \\
\text { paraprofessionals } \\
\text { Peers }\end{array}$ & $\begin{array}{c}\text { Science skills } \\
\text { (leisure skills, } \\
\text { UNO cards) } \\
\text { V }\end{array}$ & $\begin{array}{l}\text { SI, IL, TA, } \\
\text { Prompting, } \\
\text { peer tutoring } \\
\text { MPD }\end{array}$ \\
\hline Hart \& Whalon [44] & $\begin{array}{c}1 \mathrm{M} \\
\text { Southwestern } \\
\text { USA } \\
\text { ASD, IQ - }\end{array}$ & 16 & $\begin{array}{c}\text { RR, } \mathrm{T} \\
\text { paraprofessionals } \\
\text { Peers }\end{array}$ & Science skills & $\begin{array}{c}\text { Tech } \\
\text { (video modeling and } \\
\text { internet sources), } \\
\text { prompting } \\
\text { RD }\end{array}$ \\
\hline Hudson et al. [45] & $\begin{array}{l}1 \mathrm{M} 2 \mathrm{~F} \\
\text { origin- } \\
\text { ID, IQ - }\end{array}$ & - & $\begin{array}{l}\mathrm{GC}, \mathrm{T}, \mathrm{SE} \\
\text { peers }\end{array}$ & $\begin{array}{c}\text { Science skills } \\
\text { C (listening and } \\
\text { wh-questions) }\end{array}$ & $\begin{array}{l}\text { Prompting, } \\
\text { peer tutoring } \\
\text { MPD }\end{array}$ \\
\hline Jimenez et al. [46] & $\begin{array}{c}1 \mathrm{M} 2 \mathrm{~F} \\
\text { Southeastern } \\
\text { USA } \\
\text { ID, IQ: } 48-54\end{array}$ & $11-13$ & RR, T, SE & $\begin{array}{c}\text { Chemistry } \\
\text { (materials) } \\
\text { V, C } \\
\text { (KWHL diagram) }\end{array}$ & $\begin{array}{l}\text { Self-directed } \\
\text { instruction, } \\
\text { IL, TA, TD, } \\
\text { prompting } \\
\text { MPD }\end{array}$ \\
\hline Jimenez et al. [47] & $\begin{array}{c}3 \mathrm{M} 2 \mathrm{~F} \\
\text { Southeastern } \\
\text { USA } \\
\text { ID, IQ: 34-55 }\end{array}$ & $11-14$ & $\begin{array}{l}\mathrm{GC}, \mathrm{T} \\
\text { peers }\end{array}$ & $\begin{array}{c}\text { Physics } \\
\text { (kinetic energy) } \\
\text { V, C } \\
\text { (KWHL diagram) }\end{array}$ & $\begin{array}{l}\text { SI, IL, prompting, } \\
\text { TD, peer tutoring } \\
\text { MPD }\end{array}$ \\
\hline Jimenez et al. [48] & $\begin{array}{l}2 \mathrm{M} 1 \mathrm{~F} \\
\text { African } \\
\text { American } \\
\text { ASD, IQ - }\end{array}$ & 9 & $\begin{array}{c}\mathrm{RR}, \mathrm{T}, \mathrm{SE} \\
\text { paraprofessionals }\end{array}$ & $\begin{array}{c}\text { Science skills } \\
\text { (materials, life circle) } \\
\text { V, C } \\
\text { (KWHL diagram) }\end{array}$ & $\begin{array}{l}\text { IL, prompting, TD } \\
\text { MPD }\end{array}$ \\
\hline Knight et al. [49] & $\begin{array}{c}3 \mathrm{M} \\
\text { Southeastern } \\
\text { USA } \\
\text { ASD, IQ: 53-62 }\end{array}$ & $5-8$ & $\begin{array}{c}\mathrm{RR}, \mathrm{T} \\
\text { paraprofessionals }\end{array}$ & $\begin{array}{c}\text { Science skills } \\
\text { (daily life) } \\
\text { V, C } \\
\text { (inside-outside, } \\
\text { change, hot-cold, } \\
\text { same-different) }\end{array}$ & $\begin{array}{l}\text { SI, IL, prompting } \\
\text { MPD }\end{array}$ \\
\hline Knight et al. [50] & $\begin{array}{c}2 \text { M } 1 \text { F } \\
\text { Southeastern } \\
\text { USA } \\
\text { ASD, IQ: 40-55 }\end{array}$ & $13-14$ & $\begin{array}{c}\text { RR } \\
\text { Researcher }\end{array}$ & $\begin{array}{c}\text { Physics } \\
\text { (evaporation) } \\
\text { V, C } \\
\text { (graphic organizers) }\end{array}$ & $\begin{array}{c}\text { SI, TA, } \\
\text { prompting, TD } \\
\text { MPD }\end{array}$ \\
\hline
\end{tabular}


Table 1. Cont.

\begin{tabular}{|c|c|c|c|c|c|}
\hline Citation & Participants & Age (or Grade) & $\begin{array}{l}\text { Setting/ } \\
\text { Instructor }\end{array}$ & $\begin{array}{l}\text { Science Content/ } \\
\text { Teaching Targets }\end{array}$ & $\begin{array}{l}\text { Intervention } \\
\text { Design }\end{array}$ \\
\hline Knight et al. [51] & $\begin{array}{c}3 \mathrm{M} 1 \mathrm{~F} \\
\text { African } \\
\text { American } \\
\text { ASD, IQ: } 53-57\end{array}$ & $11-14$ & $\begin{array}{c}\text { RR, SE } \\
\text { Paraprofessionals }\end{array}$ & $\begin{array}{c}\text { Biology } \\
\text { (animals and energy) } \\
\text { V, C } \\
\text { (application } \\
\text { questions) }\end{array}$ & $\begin{array}{l}\text { SI, prompting } \\
\text { Tech (eText) } \\
\text { MPD }\end{array}$ \\
\hline Knight et al. [52] & $\begin{array}{c}3 \mathrm{M} 1 \mathrm{~F} \\
\text { Caucasian } \\
\text { ID, IQ: } 41-55\end{array}$ & $18-21$ & $\mathrm{RR}, \mathrm{SE}$ & $\begin{array}{c}\text { Physics } \\
\text { (forces) } \\
\text { V, C } \\
\text { (application } \\
\text { questions) }\end{array}$ & $\begin{array}{l}\text { SI, prompting } \\
\text { Tech (eText) } \\
\text { MPD }\end{array}$ \\
\hline Knight et al. [53] & $\begin{array}{c}2 \mathrm{M} 1 \mathrm{~F} \\
\text { Caucasian, } \\
\text { Hispanic } \\
1 \text { ID, } 2 \text { ASD } \\
\text { IQ: - }\end{array}$ & $7-10$ & $\begin{array}{c}\mathrm{GC}, \mathrm{T} \\
\text { paraprofessionals }\end{array}$ & $\begin{array}{l}\text { Environment } \\
\text { (geographical } \\
\text { settings) } \\
\text { V, C }\end{array}$ & $\begin{array}{c}\text { TA, prompting } \\
\text { Tech } \\
\text { (video modeling) } \\
\text { MPD }\end{array}$ \\
\hline McKissick et al. [54] & $\begin{array}{l}3 \mathrm{M} 1 \mathrm{~F} \\
\text { African } \\
\text { American and } \\
\text { Latin Hispanic } \\
\text { ASD, IQ - }\end{array}$ & $9-10$ & $\begin{array}{c}\text { RR } \\
\text { Researcher }\end{array}$ & $\begin{array}{c}\text { Science skills } \\
\text { (decision and } \\
\text { navigation skills) }\end{array}$ & $\begin{array}{l}\text { SI, prompting, TA } \\
\text { Tech (CAI) } \\
\text { MPD }\end{array}$ \\
\hline McKissick et al. [55] & $\begin{array}{c}\text { 2 M 1 F } \\
\text { Caucasian, } \\
\text { African } \\
\text { American, } \\
\text { Hispanic } \\
\text { ASD, IQ: 49-71 }\end{array}$ & $13-14$ & $\begin{array}{c}\text { RR, SE } \\
\text { Paraprofessionals }\end{array}$ & $\begin{array}{l}\text { Biology (cell) } \\
\text { V,C }\end{array}$ & $\begin{array}{l}\text { SI, prompting } \\
\text { Tech (CAI) } \\
\text { MPD }\end{array}$ \\
\hline McMahon et al. [56] & $\begin{array}{c}1 \mathrm{M} 2 \mathrm{~F} \\
\text { Southeastern } \\
\text { USA } \\
\text { ID, IQ: } 48-67\end{array}$ & $19-25$ & $\begin{array}{l}\text { University } \\
\text { Researcher }\end{array}$ & $\begin{array}{c}\text { Biology } \\
\text { (cells, body parts) } \\
\text { V, C }\end{array}$ & $\begin{array}{l}\text { SI, TA, TD, } \\
\text { Tech (AR) } \\
\text { MPD }\end{array}$ \\
\hline $\begin{array}{c}\text { Miller \& } \\
\text { Taber-Doughty [57] }\end{array}$ & $\begin{array}{c}1 \mathrm{M} 2 \mathrm{~F} \\
\text { Caucasian, Latin } \\
\text { ID, IQ: 46-63 }\end{array}$ & $12-13$ & $\begin{array}{l}\text { Kitchenette } \\
\text { Researcher }\end{array}$ & $\begin{array}{c}\text { Physics } \\
\text { (mixing material, } \\
\text { forces and motion) } \\
\text { V, C }\end{array}$ & $\begin{array}{l}\text { IL, TA } \\
\text { MPD }\end{array}$ \\
\hline Smith et al. [58] & $\begin{array}{c}3 \mathrm{M} \\
\text { Asian, African } \\
\text { American } \\
\text { ASD, IQ: 59-69 }\end{array}$ & $11-12$ & $\begin{array}{c}\mathrm{RR}, \mathrm{T} \\
\text { paraprofessionals } \\
\text { peers }\end{array}$ & $\begin{array}{c}\text { Biology (cell) } \\
\text { V } \\
\text { (Bloom taxonomy) }\end{array}$ & $\begin{array}{l}\text { SI, prompting } \\
\text { Tech (CAI) } \\
\text { MPD }\end{array}$ \\
\hline Smith et al. [59] & $\begin{array}{l}1 \mathrm{M} 2 \mathrm{~F} \\
\text { Caucasian, } \\
\text { African } \\
\text { American } \\
\text { ID, IQ: - }\end{array}$ & $6-8$ & $\begin{array}{c}\text { RR } \\
\text { Researcher }\end{array}$ & $\begin{array}{c}\text { Science skills } \\
\text { (plant and animal } \\
\text { circle life) } \\
\text { V, C } \\
\text { (KWHL diagram) }\end{array}$ & $\begin{array}{l}\text { SI, IL, TA, TD } \\
\text { MPD }\end{array}$ \\
\hline Smith et al. [60] & $\begin{array}{c}2 \mathrm{M} 1 \mathrm{~F} \\
\text { Southeastern } \\
\text { USA } \\
\text { ID, IQ: 48-65 }\end{array}$ & $22-25$ & $\begin{array}{l}\text { University } \\
\text { Researcher }\end{array}$ & $\begin{array}{l}\text { Science skills } \\
\text { (decision and } \\
\text { navigation skills) }\end{array}$ & $\begin{array}{l}\text { TA, prompting } \\
\text { Tech (AR) } \\
\text { RD }\end{array}$ \\
\hline Taylor [61] & $\begin{array}{l}1 \mathrm{M} 2 \mathrm{~F} \\
\text { Caucasian } \\
\text { ID, IQ: - }\end{array}$ & $5-7$ & $\begin{array}{c}\mathrm{RR} \\
\text { Researcher }\end{array}$ & $\begin{array}{l}\text { Science skills } \\
\text { (programming and } \\
\text { coding skills) }\end{array}$ & $\begin{array}{c}\text { SI } \\
\text { Tech (robotics) } \\
\text { MPD }\end{array}$ \\
\hline
\end{tabular}

Note: V (vocabulary), C (comprehension), M (male), F (female), RR (resource room), GC (general classroom), T (teacher), SE (special educator), SI (systematic instruction), TA (task analysis), TD (time delay), IL (inquiry learning), Tech (technology), MBD (multiple probe design), RD (reversal design). 
Table 2. Assessment of generalization, maintenance, effect sizes and social validity.

\begin{tabular}{|c|c|c|c|c|}
\hline Citation & Generalization & Maintenance & PND & Social Validity \\
\hline Britton et al. [38] & - & $\sqrt{ }$ & $54.55 \%$ & - \\
\hline Ciullo et al. [39] & - & $\sqrt{ }$ & $100 \%$ & $\sqrt{ }$ \\
\hline Collins et al. [40] & - & $\sqrt{ }$ & $84.58 \%$ & $\sqrt{ }$ \\
\hline Courtade et al. [41] & - & $\sqrt{ }$ & $96.13 \%$ & $\sqrt{ }$ \\
\hline Evmenova et al. [42] & - & - & $87.75 \%$ & $\sqrt{ }$ \\
\hline Fetko et al. [43] & - & $\sqrt{ }$ & $92.86 \%$ & - \\
\hline Hart \& Whalon [44] & - & - & $57.14 \%$ & $\sqrt{ }$ \\
\hline Hudson et al. [45] & $\sqrt{ }$ & - & $\begin{array}{l}66.80 \% \\
51.39 \% \\
\end{array}$ & $\sqrt{ }$ \\
\hline Jimenez et al. [46] & $\sqrt{ }$ & - & $\begin{array}{l}100 \% \\
100 \% \\
100 \%\end{array}$ & $\sqrt{ }$ \\
\hline Jimenez et al. [47] & - & $\sqrt{ }$ & $\begin{array}{c}94.28 \% \\
91 \% \\
75 \%\end{array}$ & $\sqrt{ }$ \\
\hline Jimenez et al. [48] & - & $\sqrt{ }$ & $\begin{array}{c}64.95 \% \\
25 \% \\
50 \%\end{array}$ & $\sqrt{ }$ \\
\hline Knight et al. [49] & $\sqrt{ }$ & - & $53.57 \%$ & $\sqrt{ }$ \\
\hline Knight et al. [50] & - & $\sqrt{ }$ & $83.33 \%$ & - \\
\hline Knight et al. [51] & - & $\sqrt{ }$ & $46.75 \%$ & $\sqrt{ }$ \\
\hline Knight et al. [52] & - & - & $90.68 \%$ & - \\
\hline Knight et al. [53] & - & $\sqrt{ }$ & $\begin{array}{c}100 \% \\
91.67 \% \\
\end{array}$ & $\sqrt{ }$ \\
\hline McKissick et al. [54] & $\sqrt{ }$ & $\sqrt{ }$ & $80.95 \%$ & $\sqrt{ }$ \\
\hline McKissick et al. [55] & - & - & $59.92 \%$ & $\sqrt{ }$ \\
\hline McMahon et al. [56] & - & - & $\begin{array}{l}96.59 \% \\
87.75 \% \\
\end{array}$ & $\sqrt{ }$ \\
\hline Miller \& Taber-Doughty [57] & $\sqrt{ }$ & - & $100 \%$ & $\sqrt{ }$ \\
\hline Smith et al. [58] & $\sqrt{ }$ & - & $100 \%$ & $\sqrt{ }$ \\
\hline Smith et al. [59] & - & $\sqrt{ }$ & $\begin{array}{c}80 \% \\
84.72 \% \\
\end{array}$ & $\sqrt{ }$ \\
\hline Smith et al. [60] & - & - & $100 \%$ & $\sqrt{ }$ \\
\hline Taylor [61] & $\sqrt{ }$ & - & $100 \%$ & - \\
\hline
\end{tabular}

\subsection{Data Synthesis}

After the coding procedure, the descriptive results were analyzed and synthesized considering similar study characteristics. Specifically, the studies are summarized in Table 1, considering the number of participants identified with ID or ASD, setting and instructor, content area for the targeted skill, intervention, and type of research design used.

\subsection{PND Effect Size}

All studies were summarized meta-analytically based on results of visual analysis of student individualized graphs. The effectiveness of intervention was determined using a nonparametric metanalytic approach [62]. Calculating the percentage of nonoverlapping 
data for each study, we recorded an overall PND for each instructional variable. We used only the data points of baseline and intervention phases because some of the studies did not present generalization or maintenance outcomes. According to Scruggs and Mastropieri [63], PND effect size can range from $0 \%$ to $100 \%$, with interpretation guidelines offered by its authors: $>70 \%$ for effective interventions, $50 \%$ to $70 \%$ for partial effectiveness, and $<50 \%$ for ineffective [64]. The two authors agreed on PND calculations.

\section{Results}

SPSS (IBM, Armonk, NY, USA) for Windows and Microsoft Excel (Microsoft Corporation, Albuquerque, NM, USA) were used to extract data. The outcomes included data extraction and comprehensive description of 24 coded studies, their synthesis, the quality analysis based on Horner's criteria and the determination of the effectiveness of the studies $[62,63]$. The studies focused on the instructional method, the supplemental practices and technology use. Table 1 presents study characteristics, participants, settings and instructors, science content, and targets. The experimental design was also noted.

\subsection{Study Characteristics}

A total of 24 studies conducted in the USA between years 2008-2019 and applied a single-case research design. In particular, 22 studies used a multiple probe across participant design and two studies used a reversal design (Table 1). All 24 studies focused on science content (targeted vocabulary and basic comprehension). The training settings included different types, such as resource rooms, inclusive classrooms, university campus, or other physical facilities in schools. The instructors were teachers, special educators, paraprofessionals, peer tutors, or researchers.

\subsection{Participants}

All 24 studies described the participants with sufficient detail and replicable precision for other researchers to recruit similar participants [28]. There was a total of 77 participants reflected across 24 documents included in this review. The studies involved from 1 to 8 participants per study between 4 to 25 years old (mean age $=12$ ). Out of those students, 50 had a diagnosis of ID and 27 ASD with ID. The participant origin is presented in Table 1, with most of them American, Hispanic, or Caucasian. All students were taught in English at school. Three of the studies do not provide information regarding student origin.

All students according to their latest evaluations had an IQ of 70 or below. The different diagnoses of ID and ASD were grouped together because of the similar academic profile of students based on the criteria of their previous reading and comprehension assessments. Additionally, all studies included gender-specific information ( 29 females and 48 males). The students were enrolled in all education levels, with most of them to be enrolled in middle school (23 students in grades K6 and K7). A total of 15 studies reported the IEP program of students.

The extracted data were relevant to the academic, communicative, and behavior content. A total of 21 studies reported information about reading, comprehension skills, functional sight words, and application questions. Most students responded very poorly to basic reading skills. Only 3 participants had extremely low or totally absent reading skills. A total of 11 studies with a total of 27 students referred to basic to high computer skills. A total of 23 studies reported student response mode (51 students responded verbally, 21 multimodal and 3 responded with other types of response, such as gestures).

Social and behavior skills were recorded in 13 studies. The outcomes indicated low to basic skills and only 5 participants reflected high level of social skills. Enough students could greet with or without prompting. Additionally, they interacted with peers and their teachers or other adults. Their behavior presented heterogenic characteristics because of their disability level. Some examples were noticed as a single behavior, such as willingness, participation, creativity, cooperation, and independence, which affected student cognitive processes (e.g., completing a task). Other students indicated negative behavior, such as 
refusal or eloping from a setting if left unattended, or repetitive ritualistic behavior, such as whining or crying when their routine was disrupted. Difficulties in academic content that students faced were extracted from 22 studies (reading, comprehension, writing, and problem-solving). Additional communicative deficits (speech delays, specifically regarding receptive and expressive language) led to limited student participation in the class.

\subsection{Setting}

Most of the studies described the setting with sufficient detail. A total of 15 studies took place in a resource or a special education classroom (7 of those followed a oneto-one instructional format), but 5 studies were conducted in a general or inclusive classroom $[41,42,45,47,53]$. Some studies took place within the tutor room or in other settings, such as college (outdoor campus) or computer lab $[56,57,60]$. The instructors were teachers, or special educators, or the researchers of each study. Specifically, teachers implemented the intervention in 8 studies and special educators in 4 studies. There were 3 studies which were carried out by teachers and special educators, too $[45,46,48]$. Researchers were involved in all 24 studies either as interventionists or as data collectors to assure interrater reliability. Paraprofessionals participated in 10 studies, and peer tutors in 6 studies.

Each study covered at least 2 phases-baseline and intervention phase. The total time duration was measured from 15 to 60 sessions. The 17 studies included a short pre-baseline phase before the students entered the baseline for them to be trained. When all of them demonstrated proficiency, based on the researcher master criteria, the baseline began. The baseline lasted from 3 to 18 sessions and the intervention phase from 4 to 19 sessions. Several studies included pauses or vacation during the implementation of intervention; however, single-case research design is considered adaptable to those conditions [28].

\subsection{Science Content and Targets}

Physics, biology, environment and science skills were included in science content aligned to the USA curriculum. The studies targeted the acquisition of grade-aligned science terms, comprehension, and application for students with ID or with ASD.

\subsection{Experimental Design}

The total of 24 studies used single-case research design with quantitative outcomes in a visual analysis of participant graphs. A functional relationship was determined between dependent and independent variables (19 studies) relevant to science vocabulary and comprehension skills to student academic achievement. The 22 studies followed a singlecase research design across participants and the other 2 used a reversal design. Visual analysis involved interpretation of the level, trend, and variability performance occurring during baseline and intervention conditions (see Table 2). Additionally, researchers of each study judged the immediacy of effects, the proportion of overlapping data points, the magnitude changes in the dependent variable, and the consistency of data patterns. The single-case design is regarded as important to determine educational interventions, as each participant serves their own control. Specifically, performance prior to intervention was compared to performance during and/or after intervention [28].

\subsection{Independent and Dependent Variable}

The independent variable in single-subject research is the intervention under investigation. We examined the articles, and we determined the instructional strategies, such as the systematic instruction and the supplemental supportive strategies that the researchers used.

Most studies (13) used systematic instruction in a total of 44 participants. Of those, 39 indicated improvement in their outcomes when PND effect sizes were calculated. The 8 studies used instructions based on inquiry skills, including 43 participants. Out of those, 37 recorded improving outcomes based on targeted skills in problem solving and in participation during the process. All these interventions were determined as effective. Just 
one study used self-directed instruction with elements of instruction based on inquiry skills, task analysis, time delay and prompting with high effectiveness based on the outcomes [46].

Analyzing the studies, we found that a combined system of teaching methods could effectively support an intervention for students with ID or/and ASD. Of the 24 instruction studies, 19 used prompting techniques and 11 studies applied task analysis. These strategies were the most frequent methods that researchers and instructors preferred. Other teaching approaches were constant time delay (7 studies) and peer tutoring (6 studies). Technology was regarded as a strong and reinforcing practice, met in 12 studies (i.e., types of technology: eText, video modeling, augmented reality, and robotics).

The dependent variables were operationally defined to allow valid and consistent assessment of the variable and replication of the assessment process [28]). The 24 singlecase research designs used 37 dependent variables defined and measured repetitively within and across controlled conditions by the number of independent correct answers or task analytic steps on probes. The outcomes were presented as an academic performance in participants' individualized graphs. The measurement of interobserver agreement (IOA) should allow assessment for each variable across each participant in each condition of the study. The 23 studies report IOA.

\subsection{Outcomes}

The interventionists and researchers measured the effects of the independent variables on the dependent variables during probe sessions. Some studies collected generalization (15) and maintenance (12) data using activity sheets to assess student use of science terms within science activities in the general education class.

Baseline phase indicates that the level of independent correct answers ranges from zero to low values, with stable or decreasing trend before intervention starts. When intervention phases began, most students indicated a change in level (immediate or not) from decreasing to stable or to increasing. In reference to data variability, there was a low or high variability, with overlapping data for interventions with partial effectiveness. In parallel, a meta-analytic procedure had occurred, documenting comparative trends in the types of appropriate instructional methods. A total of 24 studies extracted 37 dependent variables and the interventions were characterized as effective (26), partially effective (9), and ineffective (2) based on their effect size. Studies were categorized as following full stages of baseline, intervention, maintenance, and generalization.

\subsection{Experimental Control}

Regarding Horner et al. criteria [28], a total of 20 studies provided experimental control for most threats to internal validity. They allowed confirmation of a functional relationships between the manipulation of the independent variable and change in the dependent variable. A total of 2 of them provided experimental control ABAB by documenting 3 demonstrations of experimental effect at 3 different participants [44,60]. A total of 18 studies provided a similar logic for documenting experimental control for multiple baseline designs. The results documented experimental control by demonstrating a covariation between change in behavior patterns and introduction of the intervention within 3 different series at 3 different points in time. A total of 4 studies that did not demonstrate experimental control showed that the point in time coincided for all the participants $[38,46,59,61]$.

\subsection{Social Validity}

Single-subject research document interventions are functionally related to a change in socially important outcomes. Additionally, interventions report the procedures to be acceptable and feasible. Social validity was measured via questionnaires in 20 studies. Students were asked whether enjoyed participating in the study and whether they felt participating in the studies increased their knowledge of science content. Additionally, the interventionists assessed the instructor perspectives related to student ability to generalize 
skills to activities within the science classroom, the level required to deliver the intervention and their perspectives of the social importance of the intervention. As a result of this, interventions converge to the general science-teaching targets aligned to all students, for them to be included in the general science classroom.

\subsection{Interobserver Agreement (IOA)}

Interobserver agreement was taken on 21 of 24 studies (\%) including 17 of 61 participants (27.87\%). Coders agreed on 75 of 77 occurrences (97.4\%). Disagreements were fidelity of implementation and problem types. The two authors discussed differences and reached a consensus.

\subsection{Meta-Analytic Procedure}

After analyzing the studies descriptively, we investigated the characteristics of each study meta-analytically. Once we calculated the effect sizes for each individual participant across intervention, we obtained an overall ES for the intervention of each study (see Table 2). In addition, visual analysis involved interpretation of the level, trend, overlapping data, immediate change and variability of performance occurring during baseline and intervention conditions. The integration of information from these multiple assessments and comparisons was used to determine if a functional relationship exists between the independent and dependent variables. All participants increased the results in the probes in comparison to the baseline points. The generalization and maintenance phases were recorded in most of the studies in which students responded. To sum up, studies were categorized as having social validity results. Overall PND effect sizes ranged from $46.75 \%$ to $100 \%$. A total of 17 studies were effective (Table 2). When large effect sizes were calculated, the data in visual analysis showed an immediate change and an increasing trend. There was an important increase to the academic achievement compared to the low or very low level in baseline phase. The results were regarded as reliable if the performance during an intervention phase did not overlap with the performance during the baseline phase when data points were plotted over time. The outcomes of all the studies are presented at Table 2.

\section{Discussion}

Research indicates that students with ID or ASD often struggle with complex contexts regarding science teaching. Their cognitive deficits hinder full access to grade-aligned content in science classrooms. However, several adaptations and modifications may increase student participation and progress. Using the previous teaching approaches to teach science content to students with ID or ASD, students make meaningful naturalworld connections. Therefore, the aim of the present review was to explore studies of academic achievements of students with ID and ASD (depending on IQ) on science content and estimated intervention effectiveness. The specific purpose was to better understand how to improve the comprehension of basic science content. In addition, we focused on analyzing the effect sizes combined with the visual analysis of individualized graphs by determining the effectiveness of each intervention. Our review both supported and extended prior literature on science-teaching interventions for students with ID or ASD. The study highlighted that systematic instruction is an effective method of teaching academic content to students with ID or ASD [30,31,50]. Additionally, validating our findings, many other studies have found a strong relationship between science vocabulary knowledge and science comprehension especially for students who may struggle with reading skills. Additionally, previous research supported our finding that teaching the definition of science vocabulary helps students to better understand science expository text $[17,27,29,30]$.

As a result of this work, inquiry learning can be considered an effective practice when used with supplemental instructive methods [5]. Students enrolled in guided inquiry teaching programs developed social and problem-solving skills $[29,65]$. The results of the current review agree with previous research that referred to supportive methods in science teaching for students with ID or ASD. Spooner et al. [17] found that task analysis offered 
positive outcomes to student achievements. Breaking down a complicated task into steps helped students to develop attention and problem-solving skills.

A supplemental teaching technique which was met in some studies was peer tutoring or cooperative learning groups and procedural facilitation. These approaches can be used to increase comprehension for students with ID or ASD. The system of least prompts holds promise as the most popular practice in a supporting and positive learning environment. Specifically, the system of least prompts can help students to remain on task, even if work is demanding. As students with ID learn slower (because of their deficits or low patience), prompts and reinforcements are regarded appropriate and supportive during the teaching process.

Technology can benefit students with ASD and ID for them to evaluate social and communicative skills $[15,66]$. These students meet learning barriers, therefore technology related to individualized characteristics can increase participation in the classroom. Digital technology motivates students with disabilities to participate in daily activities and contributes to their academic achievements by synthesizing information in multiple ways, such as visual representations or alternative environments [2].

Using systematic instruction with supplemental practices to teach science literacy to students with ID or ASD will allow them to make more meaningful natural-world connections. The current review also extends prior reviews by including more recent research in generalization and maintenance phases during teaching process. The sufficient detailed description of participants with ID or ASD is a way to use of student strength based on their individualized needs. The teaching target-oriented knowledge usage or functional skill in daily life leads to science skill evaluation aligned with general curriculum $[6,67]$.

\section{Limitations}

Several limitations to this study suggest future research directions. First, we located a small number of studies that met the identified criteria $(n=24)$. Upon ancestral search which was conducted through the handsearching of references we included additional studies. We must notice that unpublished data from high-quality studies were not included in the review. Some keywords or other terms may not be identified in the title or abstract during database searching. One more limitation is bias against publication, which is often met in synthesis, and bias against positive findings which may be included in the review. Additionally, the results we reviewed came from USA. The absence of studies in Europe indicates the limited field in this type of research. However, it is important to recognize the contribution of the single-case research design in teaching academic content (e.g., reading, mathematics, and science) for students with ID or ASD. Researchers and practitioners should design interventions focusing on single-subject design, which is an effective research design in special education. Despite the significant difference between intervention types (systematic instruction, inquiry learning), the small number of studies made it difficult to create a meta-analytic synthesis as a combination of effect sizes. We must notice that there is still debate in the field over the use of meta-analytic techniques with single-case research designs because they neither fit a random nor a fixed effect model perfectly. Given these limitations, and the fact that research on this topic is at a nascent stage, several options for future research exist.

The researchers should discuss the results and how they can be interpreted from the perspective of previous studies and of the working hypotheses. The findings and their implications should be discussed in the broadest context possible. Future research directions may also be highlighted.

\section{Conclusions}

There is a necessity in the process of adapting effective science-teaching interventions for students with ID and ASD to be included in the general classroom. Student access to science content aligned with the general curriculum contributes to the comprehension of their individuality as a part of the natural world [34]. The use of available material 
resources, appropriate and alternative conditions, as well as the assistance of technology can support the teaching process, for students with ID or ASD to reach their full potential [68]. Science education can contribute to inclusion, providing equality for students with ID and ASD in society [69].

Author Contributions: Conceptualization, G.I. and S.-G.S.; methodology, G.I.; software, G.I.; validation, G.I. and S.-G.S.; formal analysis, G.I.; investigation, G.I.; resources, G.I.; data curation, G.I.; writing—original draft preparation, G.I.; writing—review and editing, G.I.; visualization, G.I.; supervision, S.-G.S.; project administration, S.-G.S.; All authors have read and agreed to the published version of the manuscript.

Funding: This research received no external funding.

Institutional Review Board Statement: Not applicable.

Informed Consent Statement: Not applicable.

Data Availability Statement: Not applicable.

Conflicts of Interest: The authors declare no conflict of interest.

\section{References}

1. Allor, J.H.; Mathes, P.G.; Roberts, J.K.; Cheatham, J.P.; Otaiba, S.A. Is Scientifically Based Reading Instruction Effective for Students with Below-Average IQs? Except. Child. 2014, 80, 287-306. [CrossRef]

2. Israel, M.; Wherfel, Q.M.; Pearson, J.; Shehab, S.; Tapia, T. Empowering K-12 Students With Disabilities to Learn Computational Thinking and Computer Programming. Teach. Except. Child. 2015, 48, 45-53. [CrossRef]

3. Kennedy, M.J.; Rodgers, W.J.; Romig, J.E.; Lloyd, J.W.; Brownell, M.T. Effects of a Multimedia Professional Development Package on Inclusive Science Teachers' Vocabulary Instruction. J. Teach. Educ. 2017, 68, 213-230. [CrossRef]

4. Taylor, J.; Tseng, C.; Murillo, A.; Therrien, W.; Hand, B. Using Argument-based Science Inquiry to Improve Science Achievement for Students with Disabilities in Inclusive Classrooms. J. Sci. Educ. Stud. Disabil. 2018, 21, 1-14. [CrossRef]

5. Next Generation Science Standards: For States, by States; The National Academies Press: Washington, DC, USA, 2013. Available online: https:/ / www.nap.edu/catalog/18290/next-generation-science-standards-for-states-by-states (accessed on 10 July 2021).

6. Karimi, H.; Dias, M.; Pearlman, J.; Zimmerman, G. Wayfinding and Navigation for People with Disabilities Using Social Navigation Networks. EAI Endorsed Trans. Collab. Comput. 2014, 1, e5. [CrossRef]

7. American Psychiatric Association. Diagnostic and Statistical Manual of Mental Disorders, 5th ed.; American Psychiatric Association: Washington, DC, USA, 2013. [CrossRef]

8. Andersen, L.; Nash, B. Making Science Accessible to Students with Significant Cognitive Disabilities. J. Sci. Educ. Stud. Disabil. 2016, 19, 17-38. [CrossRef]

9. Morningstar, M.E.; Kurth, J.A.; Johnson, P.E. Examining National Trends in Educational Placements for Students With Significant Disabilities. Remedial Spec. Educ. 2016, 38, 3-12. [CrossRef]

10. Odom, S.L.; Buysse, V.; Soukakou, E. Inclusion for Young Children with Disabilities: A Quarter Century of Research Perspectives. J. Early Interv. 2011, 33, 344-356. [CrossRef]

11. Algozinne, B.; Ysseldyke, J. The Fundamentals of Special Education: A Practical Guide for Every Teacher; Corwin Press: Thousand Oaks, CA, USA, 2006.

12. Westwood, P. What Teachers Need to Know about Students with Disabilities; ACER Press: Camberwell, VIC, Australia, 2009.

13. Stavroussi, P.; Papalexopoulos, P.F.; Vavougios, D. Science Education and Students with intellectual disability: Teaching Approaches and implications. Probl. Educ. 21st Century 2010, 19, 103.

14. Salmi, P. Wayfinding Design: Hidden Barriers to Universal Access. Implications 2007, 5, 1-6.

15. Root, J.R.; Stevenson, B.S.; Davis, L.L.; Geddes-Hall, J.; Test, D.W. Establishing Computer-Assisted Instruction to Teach Academics to Students with Autism as an Evidence-Based Practice. J. Autism Dev. Disord. 2017, 47, 275-284. [CrossRef] [PubMed]

16. Hopkins, S.L.; Round, P.N.; Barley, K.D. Preparing beginning teachers for inclusion: Designing and assessing supplementary fieldwork experiences. Teach. Teach. 2018, 24, 915-930. [CrossRef]

17. Spooner, F.; Knight, V.; Browder, D.; Jimenez, B.; DiBiase, W. Evaluating Evidence-Based Practice in Teaching Science Content to Students with Severe Developmental Disabilities. Res. Pract. Pers. Sev. Disabil. 2011, 36, 62-75. [CrossRef]

18. Lemons, C.J.; Allor, J.H.; Al Otaiba, S.; LeJeune, L.M. 10 Research-Based Tips for Enhancing Literacy Instruction for Students With Intellectual Disability. Teach. Except. Child. 2016, 49, 18-30. [CrossRef]

19. Mäehler, C.; Schuchardt, K. The importance of working memory for school achievement in primary school children with intellectual or learning disabilities. Res. Dev. Disabil. 2016, 58, 1-8. [CrossRef] [PubMed]

20. Anderson-Inman, L.; Horney, M.A. Supported eText: Assistive Technology through Text Transformations. Read. Res. Q. 2007, 42, 153-160. [CrossRef] 
21. Fatikhova, L.F.; Sayfutdiyarova, E.F. Improvement of Methodology of Teaching Natural Science for Students with Intellectual Disabilities by Means of 3D-Graphics. Eur. J. Contemp. Educ. 2017, 6, 229-239.

22. Grossen, B.; Carnine, D.; Romance, N.; Vitale, M. Effective strategies for teaching science. In Effective Teaching Strategies That Accommodate Diverse Learners, 4th ed.; Coyne, M.D., Kame'enui, E.J., Carnine, D.W., Eds.; Pearson Education: Upper Saddle River, NJ, USA, 2011; pp. 181-212.

23. American Association for Advancement of Science. Benchmarks for Science Literacy. Project 2061 AAAS Updates. 2009. Available online: http:/ / www.project2061.org/publications/bsl/online/index.php (accessed on 11 July 2021).

24. Scruggs, T.E.; Mastropieri, M.A. Classroom applications of mnemonic instruction: Acquisition, maintenance, and generalization. Except. Child. 1992, 58, 219-229. [CrossRef]

25. Scruggs, T.E.; Mastropieri, M.A. The construction of scientific knowledge by students with mild disabilities. J. Spec. Educ. 1994, 28, 307-321. [CrossRef]

26. Spooner, F.; McKissick, B.R.; Knight, V.F. Establishing the State of Affairs for Evidence-Based Practices in Students With Severe Disabilities. Res. Pract. Pers. Sev. Disabil. 2017, 42, 8-18. [CrossRef]

27. Courtade, G.R.; Spooner, F.; Browder, D.M. Review of Studies With Students With Significant Cognitive Disabilities Which Link to Science Standards. Res. Pract. Pers. Sev. Disabil. 2007, 32, 43-49. [CrossRef]

28. Horner, R.H.; Carr, E.G.; Halle, J.; McGee, G.; Odom, S.; Wolery, M. The Use of Single-Subject Research to Identify Evidence-Based Practice in Special Education. Except. Child. 2005, 71, 165-179. [CrossRef]

29. Rizzo, K.; Taylor, J. Effects of Inquiry-Based Instruction on Science Achievement for Students with Disabilities: An Analysis of the Literature. J. Sci. Educ. Stud. Disabil. 2016, 10, 1-16.

30. Apanasionok, M.M.; Hastings, R.P.; Grindle, C.F.; Watkins, R.C.; Paris, A. Teaching science skills and knowledge to students with developmental disabilities: A systematic review. J. Res. Sci. Teach. 2019, 56, 847-880. [CrossRef]

31. Knight, V.F.; Wood, L.; McKissick, B.R.; Kuntz, E.M. Teaching Science Content and Practices to Students with Intellectual Disability and Autism. Remedial Spec. Educ. 2020, 41, 327-340. [CrossRef]

32. Taylor, J.C.; Rizzo, K.L.; Hwang, J.; Hill, D. A review of research on science instruction for students with autism spectrum disorder. Sch. Sci. Math. 2020, 120, 116-125. [CrossRef]

33. Olson, A.; Leko, M.M.; Roberts, C.A. Providing Students With Severe Disabilities Access to the General Education Curriculum. Res. Pract. Pers. Sev. Disabil. 2016, 41, 143-157. [CrossRef]

34. Miller, B. Ensuring Meaningful Access to the Science Curriculum for Students with Significant Cognitive Disabilities. Teach Except. Child. 2012, 44, 16-25. [CrossRef]

35. Mulvey, B.K.; Chiu, J.L.; Ghosh, R.; Bell, R.L. Special education teachers' nature of science instructional experiences. J. Res. Sci. Teach. 2016, 53, 554-578. [CrossRef]

36. Moher, D.; Liberati, A.; Tetzlaff, J.; Altman, D.G.; Group, T.P. Preferred Reporting Items for Systematic Reviews and Meta-Analyses: The PRISMA Statement. PLoS Med. 2009, 6. [CrossRef] [PubMed]

37. Li, F. Research Synthesis and Meta-Analysis: A Step-by-Step Approach, 5th ed.; Cooper, H., Ed.; Sage Publications, Inc.: Thousand Oaks, CA, USA, 2019; Volume xviii, p. 360.

38. Britton, N.S.; Collins, B.C.; Ault, M.J.; Bausch, M.E. Using a Constant Time Delay Procedure to Teach Support Personnel to Use a Simultaneous Prompting Procedure. Focus Autism Other Dev. Disabl. 2015, 32, 102-113. [CrossRef]

39. Ciullo, S.; Falcomata, T.S.; Pfannenstiel, K.; Billingsley, G. Improving Learning With Science and Social Studies Text Using Computer-Based Concept Maps for Students With Disabilities. Behav. Modif. 2014, 39, 117-135. [CrossRef]

40. Collins, B.C.; Terrell, M.; Test, D.W. Using a Simultaneous Prompting Procedure to Embed Core Content When Teaching a Potential Employment Skill. Career Dev. Transit. Except. Individ. 2017, 40, 36-44. [CrossRef]

41. Courtade, G.R.; Browder, D.M.; Spooner, F.; DiBiase, W. Training Teachers to Use an Inquiry-Based Task Analysis to Teach Science to Students with Moderate and Severe Disabilities. Educ. Train. Autism Dev. Disabil. 2010, 45, 378-399.

42. Evmenova, A.S.; Graff, H.J.; Behrmann, M.M. Providing Access to Academic Content for High-School Students with Significant Intellectual Disability Through Interactive Videos. Focus Autism Other Dev. Disabl. 2015, 32, 18-30. [CrossRef]

43. Fetko, E.E.; Collins, B.C.; Hager, K.D.; Spriggs, A.D. Embedding Science Facts in Leisure Skill Instruction Conducted by Peer Tutors. Educ. Train. Autism Dev. Disabil. 2013, 48, 400-411.

44. Hart, J.E.; Whalon, K.J. Using Video Self-Modeling Via iPads to Increase Academic Responding of an Adolescent with Autism Spectrum Disorder and Intellectual Disability. Educ. Train. Autism Dev. Disabil. 2012, 47, 438-446.

45. Hudson, M.E.; Browder, D.M.; Jimenez, B.A. Effects of a Peer-Delivered System of Least Prompts Intervention and Adapted Science Read-Alouds on Listening Comprehension for Participants with Moderate Intellectual Disability. Educ. Train. Autism Dev. Disabil. 2014, 49, 60-77.

46. Jimenez, B.A.; Browder, D.M.; Courtade, G.R. An Exploratory Study of Self-Directed Science Concept Learning by Students with Moderate Intellectual Disabilities. Res. Pract. Pers. Sev. Disabil. 2009, 34, 33-46. [CrossRef]

47. Jimenez, B.A.; Browder, D.M.; Spooner, F.; Dibiase, W. Inclusive Inquiry Science Using Peer-Mediated Embedded Instruction for Students with Moderate Intellectual Disability. Except. Child. 2012, 78, 301-317. [CrossRef]

48. Jimenez, B.; Lo, Y.; Saunders, A. The Additive Effects of Scripted Lessons Plus Guided Notes on Science Quiz Scores of Students with Intellectual Disability and Autism. J. Spec. Educ. 2014, 47, 231-244. [CrossRef] 
49. Knight, V.F.; Smith, B.R.; Spooner, F.; Browder, D. Using explicit instruction to teach science descriptors to students with autism spectrum disorder. J. Autism Dev. Disord. 2012, 42, 378-389. [CrossRef] [PubMed]

50. Knight, V.F.; Spooner, F.; Browder, D.M.; Smith, B.R.; Wood, C.L. Using Systematic Instruction and Graphic Organizers to Teach Science Concepts to Students With Autism Spectrum Disorders and Intellectual Disability. Focus Autism Other Dev. Disabl. 2013, 28, 115-126. [CrossRef]

51. Knight, V.F.; Wood, C.L.; Spooner, F.; Browder, D.M.; O’Brien, C.P. An Exploratory Study Using Science eTexts With Students With Autism Spectrum Disorder. Focus Autism Other Dev. Disabl. 2014, 30, 86-99. [CrossRef]

52. Knight, V.F.; Creech-Galloway, C.E.; Karl, J.M.; Collins, B.C. Evaluating Supported eText to Teach Science to High School Students With Moderate Intellectual Disability. Focus Autism Other Dev. Disabl. 2017, 33, 227-236. [CrossRef]

53. Knight, V.F.; Kuntz, E.M.; Brown, M. Paraprofessional-Delivered Video Prompting to Teach Academics to Students with Severe Disabilities in Inclusive Settings. J. Autism Dev. Disord. 2018, 48, 2203-2216. [CrossRef] [PubMed]

54. McKissick, B.; Spooner, F.; Wood, C.; Diegelmann, K. Effects of computer-assisted explicit instruction on map-reading skills for students with autism. Res. Autism Spectr Disord. 2013, 7, 1653-1662. [CrossRef]

55. McKissick, B.R.; Davis, L.L.; Spooner, F.; Fisher, L.B.; Graves, C. Using Computer-Assisted Instruction to Teach Science Vocabulary to Students With Autism Spectrum Disorder and Intellectual Disability. Rural Spec. Educ. Q. 2018, 37, 207-218. [CrossRef]

56. McMahon, D.D.; Cihak, D.F.; Wright, R.E.; Bell, S.M. Augmented Reality for Teaching Science Vocabulary to Postsecondary Education Students With Intellectual Disabilities and Autism. J. Res. Technol. Educ. 2016, 48, 38-56. [CrossRef]

57. Miller, B.; Taber-Doughty, T. Self-Monitoring Checklists for Inquiry Problem-Solving: Functional Problem-Solving Methods for Students with Intellectual Disability. Educ. Train. Autism Dev. Disabil. 2014, 49, 555-567.

58. Smith, B.; Spooner, F.; Wood, C. Using embedded computer-assisted explicit instruction to teach science to students with autism spectrum disorder. Res. Autism Spectr. Disord. 2013, 7, 433-443. [CrossRef]

59. Smith, B.R.; Spooner, F.; Jimenez, B.A.; Browder, D. Using an Early Science Curriculum to Teach Science Vocabulary and Concepts to Students with Severe Developmental Disabilities. Educ. Treat. Child. 2013, 36, 1-31. [CrossRef]

60. Smith, C.C.; Cihak, D.F.; Kim, B.; McMahon, D.D.; Wright, R. Examining Augmented Reality to Improve Navigation Skills in Postsecondary Students With Intellectual Disability. J. Spec. Educ. Technol. 2016, 32, 3-11. [CrossRef]

61. Taylor, M.S. Computer Programming with Pre-K Through First-Grade Students With Intellectual Disabilities. J. Spec. Educ. 2018, 52, 78-88. [CrossRef]

62. Scruggs, T.E.; Mastropieri, M.A.; Casto, G. The quantitative synthesis of single-subject research: Methodology and validation. RASE Remedial Spec. Educ. 1987, 8, 24-33. [CrossRef]

63. Scruggs, T.E.; Mastropieri, M.A. How to Summarize Single-Participant Research: Ideas and Applications. Exceptionality 2001, 9 , 227-244. [CrossRef]

64. Scruggs, T.E.; Mastropieri, M.A. Summarizing Single-Subject Research: Issues and Applications. Behav. Modif. 1998, 22, 221-242. [CrossRef]

65. Scruggs, T.E.; Mastropieri, M.A.; McDuffie, K.A. Co-Teaching in Inclusive Classrooms: A Metasynthesis of Qualitative Research. Except. Child. 2007, 73, 392-416. [CrossRef]

66. Villanueva, M.; Taylor, J.; Therrien, W.; Hand, B. Science education for students with special needs. Stud. Sci. Educ. 2012, 48, 187-215. [CrossRef]

67. Lynch, S.; Taymans, J.; Watson, W.A.; Ochsendorf, R.J.; Pyke, C.; Szesze, M.J. Effectiveness of a Highly Rated Science Curriculum Unit for Students with Disabilities in General Education Classrooms. Except. Child. 2007, 73, 202-223. [CrossRef]

68. Ballard, S.L.; Dymond, S.K. Addressing the General Education Curriculum in General Education Settings with Students with Severe Disabilities. Res. Pract. Pers. Sev. Disabil. 2017, 42, 155-170. [CrossRef]

69. Millar, R. Twenty First Century Science: Insights from the Design and Implementation of a Scientific Literacy Approach in School Science. Int. J. Sci. Educ. 2006, 28, 1499-1521. [CrossRef] 